\title{
Prospects for first heavy ion physics with the ALICE experiment
}

\author{
Massimo Masera, for the ALICE Collaboration \\ Dipartimento di Fisica Sperimentale dell'Univertsità and I.N.F.N., via P. Giuria 1, 10125 Torino, Italy
}

\begin{abstract}
The ALICE experiment is the only one at the LHC which has been specifically designed for the study of nuclear collisions. Thanks to the extensive experience gained during the pp running of 2009 and 2010, the experiment is now fully commissioned and ready to make optimum use of the first nuclear collisions at the end of 2010. This talk will present a summary of the Physics potential of ALICE with Heavy Ions with a specific emphasis on the goals of the first run.
\end{abstract}

Keywords: Ultra-relativistic Heavy Ion Collisions, LHC

\section{Introduction}

The purpose of ultra-relativistic heavy-ion collision experiments is to create in the laboratory an enviroment with extremely high energy density in order to form and study the Quark-Gluon Plasma (QGP), a state of strongly interacting matter, predicted by the Quantum Chromo-Dynamics (QCD) theory, in which quarks and gluons are no longer confined within hadrons. The $\mathrm{Pb}-\mathrm{Pb}$ collisions programme starting in November 2010 at the Large Hadron Collider represents a major step forward with respect to the conditions reached at the SPS and then at the Relativistic Heavy Ion Collider (RHIC) as far as the energy density, the temperature and the QGP lifetime are concerned. The centre-of-mass energy per nucleon pair for the first run is $\sqrt{s_{N N}}=2.76 \mathrm{TeV}$, that, even though it is one half of the target value, exceeds by more than one order of magnitude the colliding energy reached so far. ALICE (A Large Ion Collider Experiment) [1] is specifically designed as a general purpose experiment for nucleus-nucleus collisions to allow a comprehensive study of both soft and hard probes related to the onset of a deconfined phase of strongly interacting matter. The apparatus has been designed to cover a broad range of phenomena which were addressed in the previous heavy-ion facilities by different dedicated experiments. ALICE has excellent vertexing capabilities and, thanks to low material budget detectors and a $0.5 \mathrm{~T}$ solenoidal magnetic field, is able to reconstruct and identify charged particles in its central barrel $\left(-0.9<\eta<0.9\right.$ with full azimuthal coverage) down to low $p_{T}$. Muon reconstruction is performed by a dedicated muon spectrometer in the forward region $(-2.5<\eta<4.0)$ and the centrality determination is done by means of zero degrees calorimeters. The $2010 \mathrm{~Pb}-\mathrm{Pb}$ run will be carried out with the same detector configuration used for proton-proton collisions: the installation of the calorimeters and of the Transition Radiation Detector (TRD) will be completed after 2010. The $2010 \mathrm{~Pb}-\mathrm{Pb}$ run is expected to last one month with a luminosity $L_{0}=10^{25} \mathrm{~cm}^{-2} \mathrm{~s}^{-1}$, two orders of magnitude below the nominal value. This is due to the reduced number of bunches circulating in the machine and to the reduced focusing with respect to the design value $\left(\beta^{*}=0.5 \mathrm{~m}\right)$ foreseen for full energy runs at $\sqrt{s_{N N}}=5.5 \mathrm{TeV}$. The initial interaction rate for minimum bias collisions will be $50 \div 100 \mathrm{~Hz}$. The expected data sample for the whole run will be $\sim 1 \div 3 \mu \mathrm{b}^{-1}$ corresponding to $0.8 \div 2.410^{7}$ minimum bias events. For the first run it will be important to sample the whole cross section down to peripheral events, applying triggers with no bias. The main trigger detectors in the barrel will be the Silicon Pixel Detector (SPD) and the V0 detector. The muon 
arm will provide an independent trigger based on Resistive Plate Chambers. The SPD is the innermost part of the Inner Tracking System (ITS). It consists of two cylindrical layers of pixel detectors located at radial distances of $3.9 \mathrm{~cm}$ and $7.6 \mathrm{~cm}$ from the beam axis. The SPD pseudorapidity acceptance is $|\eta|<2$ and $|\eta|<1.4$ for the first and second layers respectively. The V0 detector consists of two scintillator arrays placed at distances $z=3.3 \mathrm{~m}$ (V0-A, $2.8<\eta<5.1)$ and $z=-0.9 \mathrm{~m}(\mathrm{~V} 0-\mathrm{C},-3.7<\eta<-1.7)$. The V0 provides both amplitude and time information, the latter with a resolution of $1 \mathrm{~ns}$, allowing upstream collisions rejection. The physics goals of the first $\mathrm{LHC} \mathrm{Pb}-\mathrm{Pb}$ run should be established at the light of the actually expected sample size; for comparison sake, the ALICE target integrated luminosity was $\mathcal{L}=0.5 \mathrm{nb}^{-1}$ to collect enough statistics for relatively low cross section probes like quarkonia, heavy flavours and high energy jets.

\section{First Pb-Pb physics}

The first physics results will concern the global features of $\mathrm{Pb}-\mathrm{Pb}$ collisions and then observables related to soft processes. The actual transverse momentum reach will depend on the collected statistics. In this section few examples of physics issues that should be well addressed in the first heavy ion run at the LHC will be given. The first three examples concern topics that are likely to produce results in a very short time scale. The others will require more elaborate analyses and will benefit by the availability of the whole data sample. The first measurement will be the charged-particle pseudorapidity density, which is related to the energy density and the temperature reached in the interaction and for this reason is a parameter that should be fixed from the data to obtain predictions for most of the other observables. The estimates currently available for collisions with a given number of participant nucleons $\left(N_{\text {part }}=350\right)$, based on models that give a correct description of the particle production at RHIC, are mostly in the broad range $1000<d N_{c h} / d \eta<2000$, even though both lower and higher multiplicity scenarios are not excluded [2]. The measurement of the multiplicity density will be accomplished during the very first days of data taking since it can be done with some $10^{5}$ triggered events, using the information provided by few sub-systems: the SPD, the V0 and the Zero Degree Calorimeters (ZDC). The measurement is based on the number of reconstructed tracklets, that are built by pairing two hits on the two SPD layers that satisfy a selection criterion based on azimuthal and polar angles of the two hits, using the measured primary vertex position as the origin. Samples with defined centrality will be selected by means of the V0 and ZDC detectors. The use of the SPD tracklets provides a coverage of roughly 3 units of pseudorapidity. If also the information from the Forward Multiplicity Detector (FMD) is used, the coverage is extended to $\sim 8$ units of pseudorapidity.
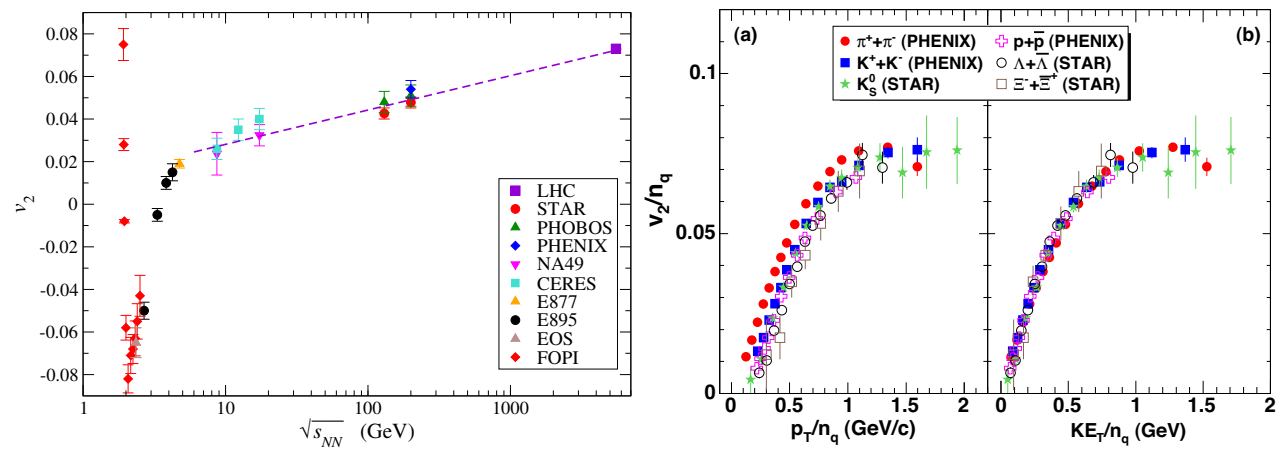

Figure 1: Left: integrated $v_{2}$ as a function of $\sqrt{s_{N N}}(y=0)$ in mid-central collisions [3]. Right: (a) $v_{2} / n_{q}$ vs $p_{T} / n_{q}$ and (b) $v_{2} / n_{q}$ vs $K E_{T} / n_{q}$ for identified particle species obtained in minimum bias Au+Au collisions. $n_{q}$ is the number of constituent quarks. [4].

Another important measurement which can be done in the first days of data taking is the evaluation of the azimuthal anisotropy of the particle production. When nuclei collide at finite impact parameter, the overlap region is almond shaped in the transverse plane. If the matter produced in the collision is interacting, the spatial asymmetry is converted 
into an anisotropic momentum distribution via multiple collisions. The azimuthal dependence of the particle yield can be written as a Fourier expansion:

$$
E \frac{d^{3} N}{d^{3} p}=\frac{1}{2 \pi} \frac{d^{2} N}{p_{T} d p_{t} d y}\left\{1+\sum_{n=1}^{\infty} 2 v_{n} \cos \left[n\left(\phi-\psi_{R}\right)\right]\right\}
$$

where $\psi_{R}$ is the angle formed by the plane defined by the $z$ axis and the impact parameter (reaction plane) with the $x z$ plane. The anisotropy is expressed by the second coefficient $v_{2}=\left\langle\cos \left[2\left(\phi-\psi_{R}\right)\right]\right\rangle$ that is called elliptic flow. A positive value of $v_{2}$ indicates an excess of particle production in the reaction plane. The large elliptic flow observed at RHIC can be accounted for by models based on relativistic hydrodynamics with a QGP equation of state and vanishing viscosity [5]. In Fig.1, left panel, a compilation of integrated $v_{2}$ data is shown as a function of $\sqrt{s_{N N}}$. The LHC point is a linear extrapolation from SPS and RHIC data. The present expectations for the LHC are for a large $v_{2}$, with an increase with respect to the RHIC data, that ranges from $10 \%$ to $50 \%$. In particular models which do not introduce corrections (e.g. [5]) for a non zero shear viscosity tend to predict elliptic flow values in the lower range. Several phenomena, like jets, can contribute to the measured anisotropy, hence several methods to extract $v_{2}$ from the data, as the 2- an 4-particle cumulant methods [6], with different sensitivities to non flow effects have been implemented in the ALICE analysis software. The flow measurement will be carried out initially by using the tracking systems of the barrel (ITS and the Time Projection Chamber - TPC) and the V0 detector. The $v_{2}$ coefficient will be determined in centrality classes and as a function of $p_{T}$.

A third early measurement will concern the so called nuclear modification factor $R_{A A}$ for the charged particles reconstructed in the ALICE barrel. The partons generated in $\mathrm{Pb}-\mathrm{Pb}$ collisions are expected to interact with the medium, which is estremely hot and dense. The resulting energy loss explains the observed suppression of high $p_{T}$ hadrons, which are produced through the fragmentation of high $p_{T}$ partons, formed in hard processes in the early phases of the collisions. This effect can be quantified by the $R_{A A}$ parameter, which is defined as the ratio of the charged particle yield in $\mathrm{Pb}-\mathrm{Pb}$ collisions to that in $\mathrm{pp}$, scaled by the number of binary nucleon-nucleon collisions $\left\langle N_{\text {coll }}\right\rangle$ :

$$
R_{A A}\left(p_{T}\right)=\frac{\left(1 / N_{e v t}^{A A}\right) d^{2} N_{\mathrm{ch}}^{A A} / d \eta d p_{T}}{\left\langle N_{\mathrm{coll}}\right\rangle\left(1 / N_{e v t}^{p p}\right) d^{2} N_{\mathrm{ch}}^{p p} / d \eta d p_{T}},
$$

Any deviation from $R_{A A}=1$ indicates that nuclear collisions are not simply a superposition of binary collisions between nucleons. An important source of systematic errors will be the pp reference, since there are no available data at $\sqrt{s_{N N}}=2.76 \mathrm{TeV}$ and it is necessary to rely on appropriate interpolations of pp data at $\sqrt{s_{N N}}=900 \mathrm{GeV}$ and $\sqrt{s_{N N}}=7 \mathrm{TeV}$.
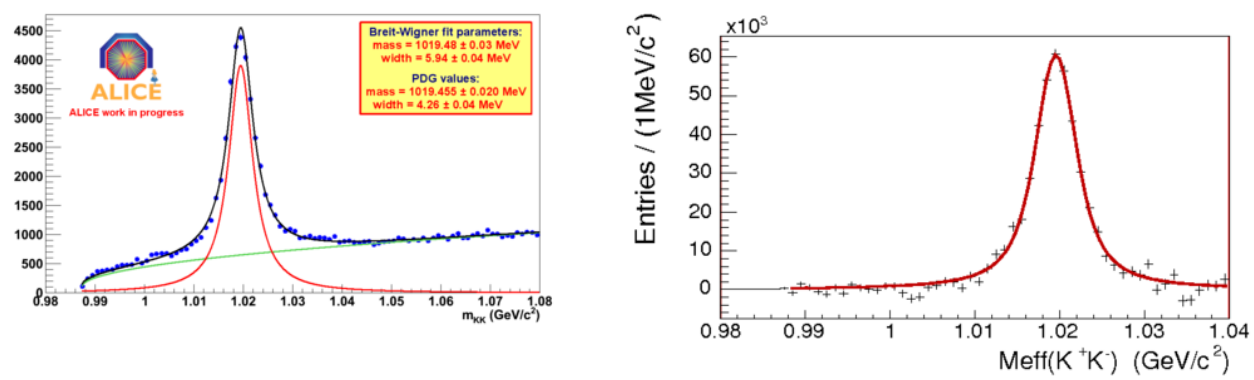

Figure 2: Left: Performance of $\phi \rightarrow K^{+} K^{-}$reconstruction for pp $7 \mathrm{TeV}$ data. Fits to the combinatorial background $(B)$ and to the signal $(S)$ are shown. Right: Expected performance of $\phi \rightarrow K^{+} K^{-}$reconstruction for $\mathrm{Pb}-\mathrm{Pb}$ data $\left(\sqrt{s_{N N}}=5.5 \mathrm{TeV}\right.$ and $\left.p_{T} \geq 2.2 \mathrm{GeV} / c\right)$. A good kaon identification with the barrel detectors is needed to reduce the large combinatorial background, which is fitted and then subtracted. The expected significance for this sample is $S / \sqrt{(S+B)}=108$ [8].

The good acceptance of ALICE at low transverse momentum and the good particle identification capabilities in the central rapidity region will allow to measure $p_{T}$ spectra, the $R_{A A}$ for identified particle species and to determine 
particle yields and ratios. The statistical models provide accurate predictions [7] for particle ratios, for the freeze-out temperature, $T^{L H C}=161 \pm 4 \mathrm{MeV}$, and for the baryo-chemical potential, $\mu_{B}^{L H C}=0.8_{0.6}^{1.2} \mathrm{MeV}$. The analyses on identified particles with pp data are well under way and the same analysis tools will be exploited for $\mathrm{Pb}-\mathrm{Pb}$ as well. As an example, in Fig.2, left panel, the reconstruction capability of the $\phi$ meson in its $\phi \rightarrow K^{+} K^{-}$decay channel is shown for $7 \mathrm{TeV}$ pp data. In the right panel, the expected performance for $\mathrm{Pb}-\mathrm{Pb}$ data is shown. For a sample of $10^{7}$ events, corresponding, as an order of magnitude, to the whole $2010 \mathrm{~Pb}-\mathrm{Pb}$ sample, the $p_{T}$ reach for $\phi, K$ and $\Lambda$ is up to $13 \div 15 \mathrm{GeV} / \mathrm{c}$, while for $\rho, \Xi$ and $\Omega$ is up to $9 \div 12 \mathrm{GeV} / \mathrm{c}$. Also the azimuthal anysotropy will be measured for identified particles, allowing a determination of $v_{2}$ for strange particles up to $p_{T} \sim 10 \mathrm{Gev} / \mathrm{c}$ for the whole sample. In Fig. 1, the right panel presents an important result obtained at RHIC: the elliptic flow per constituent quark shows an excellent scaling over the full range of transverse kinetic energy divided by the number of contituent quarks $\left(K E T / n_{q}\right)$. This behaviour, that will be checked at the LHC energy, is interpreted as an indication of the inherent quark-like degrees of freedom in the flowing matter.

ALICE will study the jet production in the central region by means of the tracking detectors, since the electromagnetic calorimeter is not presently fully installed. One of the most interesting results obtained at RHIC was the outcome of the azimuthal correlation studies of jets: a strong suppression of the away-side jet was observed [9]. This study should be feasible with the ALICE detector with a sample of $\sim 10^{6}$ central $\mathrm{Pb}-\mathrm{Pb}$ collisions, that is the order of magnitude of the foreseen data sample of 2010. We expect to collect $\sim 100$ jets with $E_{T}>100 \mathrm{GeV}$, that will allow first glimpse to high $p_{T}$ phenomena. The study of quarkonia states is extremely important to assess the properties of the medium formed in the collision. ALICE detects quarkonia both in the $e^{+} e^{-}$and $\mu^{+} \mu^{-}$decay channels respectively at central $(-0.9<\eta<0.9)$ and forward $(-4.0<\eta<-2.5)$ rapidities. The quarkonia detection performance has been tested with pp data: both decay channels can be exploited even though the TRD to identify high momentum electrons is not completely installed for this run. The overall performance depends critically on the integrated luminosity: for $\mathcal{L}=2 \mu \mathrm{b}^{-1}$, a few $10^{3} \mathrm{~J} / \psi$ will be detected and a few tens of $\Upsilon$. The $\psi^{\prime}$ will be marginal. The extrapolation from charmonium results at lower energy is not trivial, because there could be a complex interplay between melting (of $\chi_{c}$ and $\psi^{\prime}$ and possibly of $J / \psi$ ) and $c \bar{c}$ recombination. In this respect, the results of 2010 may not be conclusive. With the pp data collected this, ALICE demonstrated its good potential also to detect open heavy flavours, in particular to fully reconstruct $D$ mesons in their hadronic decay channels [10]. The estimated relative statistical error for $D^{0} \rightarrow K^{-} \pi^{+}$is below $10 \%$ for $p_{T} \lesssim 10 \mathrm{GeV} / \mathrm{c}$ for $10^{7}$ central $\mathrm{Pb}-\mathrm{Pb}$ events (centrality class: $0-10 \%$ ). If the 2010 data will consist $10^{6}$ central events, the errors will be three times higher. Hence also for open charm studies the dependence on the integrated luminosity is crucial.

\section{Conclusions}

The 2010 LHC Heavy Ion programme will allow to address most of the physics topics involving soft QCD processes and will provide results with a limited statistics for hard QCD observables. At the time of writing these notes, the first results are already available: they concern the charged multiplicity density [11] and the dependence of the multiplicity on the centrality [12], the elliptic flow of charged particles [13] and a measure of the nuclear modification factor charged particles [14].

\section{References}

[1] K. Aamodt et al. (ALICE), J. Instrum. 3, S08002 (2008).

[2] N. Armesto, arXiv:0903.1330v2 [hep-ph] (2010).

[3] N. Borghini and U. Wiedemann, J. Phys. G35, 023001 (2008).

[4] A. Adare et al. , Phys. Rev. Lett. 98, 162301 (2007).

[5] G. Kenstin and U.W. Heinz, Eur. Phys. J. C 61, 545 (2009).

[6] N. Borghini et al. , Phys. Rev. C 64, 054901 (2001).

[7] S. Abreu et al., J. Phys. G 35, 054001 (2008).

[8] B. Alessandro et al. , J. Phys. G 32, 1295 (2006).

[9] Adams et al. , Phys. Rev. Lett. 91, 072304 (2003).

[10] A. Dainese for the ALICE Collaboration, these proceedings.

[11] K. Aamodt et al. (ALICE), arXiv:1011.3916v2 [nucl-ex] (2010).

[12] K. Aamodt et al. (ALICE), arXiv:1012.1657v1 [nucl-ex] (2010).

[13] K. Aamodt et al. (ALICE), arXiv:1011.3914v1 [nucl-ex] (2010).

[14] K. Aamodt et al. (ALICE), arXiv:1012.1004v1 [nucl-ex] (2010). 